\title{
Some Differential Inequalities on Time Scales and Their Applications to Feedback Control Systems
}

\author{
Yonghong Fan, Yangyang Yu, and Linlin Wang \\ School of Mathematics and Statistics Science, Ludong University, Yantai, Shandong 264025, China \\ Correspondence should be addressed to Linlin Wang; wangll_1994@sina.com
}

Received 30 August 2016; Revised 21 December 2016; Accepted 10 January 2017; Published 28 February 2017

Academic Editor: Rigoberto Medina

Copyright (C) 2017 Yonghong Fan et al. This is an open access article distributed under the Creative Commons Attribution License, which permits unrestricted use, distribution, and reproduction in any medium, provided the original work is properly cited.

\begin{abstract}
This paper deals with feedback control systems on time scales. Firstly, we generalize the semicycle concept to time scales and then establish some differential inequalities on time scales. Secondly, as applications of these inequalities, we study the uniform ultimate boundedness of solutions of these systems. We give a new method to investigate the permanence of ecosystem on time scales. And some known results have been generalized. Finally, an example is given to support the result.
\end{abstract}

\section{Introduction}

The theory of time scales was introduced by Hilger in his Ph.D. thesis [1] in 1988. The study of dynamic equations on time scales has recently attracted a lot of attention, because it reveals many discrepancies and helps to avoid proving results twice, for differential equations and difference equations, respectively. The time scales calculus has wide applications in various fields like biology, engineering, economics, physics, neural networks, social sciences, and so on [2].

On the other hand, the permanence of a system is very important. As we know, permanence is affected by such factors as environment, food supplies, attack rates, and so on. As an example, Schreiber and Patel [3] showed that ecoevolutionary feedbacks can mediate permanence at intermediate trade-offs in the attack rates. So it is worthwhile considering the permanence of biological models. The permanence of biological systems has been discussed by many authors [417]. In the process of studying these problems, the comparison method plays a paramount role. Such as in $[4,6]$, some sufficient conditions of permanence for biological systems were established by the comparison method. Permanence is also applied in physics, chemistry, physiology, diseases, economics, and so on. Such as in [18], sufficient conditions of the permanence for a SEIRS system were obtained.
To the best of our knowledge, the permanence of ecosystem on time scales with feedback control was first investigated in [13]. The authors considered the $\mathrm{N}$-species cooperation system with time delays and feedback control

$$
\begin{aligned}
& x_{i}^{\Delta}(t)=r_{i}(t)\{1 \\
& -\frac{\exp \left\{x_{i}\left(t-\tau_{i i}\right)\right\}}{k_{i}(t)+\sum_{j=1, j \neq i}^{n} e_{i j}(t) \exp \left\{x_{j}\left(t-\tau_{i j}\right)\right\}} \\
& \left.-c_{i}(t) \exp x_{i}\left(t-\tau_{i i}\right)\right\}-d_{i}(t) y_{i}(t)-h_{i}(t) y_{i}(t \\
& \left.-\eta_{i}\right), \\
& y_{i}^{\Delta}(t)=-a_{i}(t) y_{i}(t)+b_{i}(t) \exp \left\{x_{i}(t)\right\}+\beta_{i}(t) \\
& \cdot \exp \left\{x_{i}\left(t-\sigma_{i}\right)\right\},
\end{aligned}
$$

where $i=1,2, \ldots, n$, with the initial condition

$$
x_{i}(\theta), y_{i}(\theta) \geq 0 \text { are all rd-continuous, } \theta \in\left[-\tau, t_{0}\right) \cap \mathbb{T}, x_{i}\left(t_{0}\right), y_{i}\left(t_{0}\right)>0, i=1,2, \ldots, n \text {, }
$$


on time scales $\mathbb{T}$ (which is $p$-periodic; i.e., there exits $p>0$ such that if $t \in \mathbb{T}$; then $t+p \in \mathbb{T}$ ), where $\Delta$ stands for the delta-derivative and $1-\mu(t) a_{i}(t)>0, i=1,2, \ldots, n$, as $\mu(t)=$ $\sigma(t)-t, \sigma(t)$ is defined in the second section (Definition 8).

For system (1), they assumed the following:

(H1) $r_{i}(t), a_{i}(t), b_{i}(t), c_{i}(t), d_{i}(t), e_{i j}(t), h_{i}(t), k_{i}(t)$, and $\beta_{i}(t)(i, j=1,2, \ldots, n)$ are all rd-continuous, real-valued functions which are bounded above and below by positive constants; $\tau_{i j}, \eta_{i}, \sigma_{i} \in \mathbb{T}_{p}$ $(i, j=1,2, \ldots, n)$ are positive constants. $\tau=$ $\max \left\{\max _{1 \leq i, j \leq n} \tau_{i j}, \max _{1 \leq i \leq n} \sigma_{i}, \max _{1 \leq i \leq n} \eta_{i}\right\}>0$, where $\mathbb{T}_{p}$ can be found in [13].

(H2) $-r_{i}^{l} c_{i}^{l}, r_{i}^{l}\left(k_{i}^{-1}\right)+c_{i}^{u} \in \mathscr{R}^{+}=\{p \in \mathscr{R}, 1+\mu(t) p(t)>$ 0 forall $t \in \mathbb{T}\}(i=1,2, \ldots, n)$ are all positively regressive ( $\mathscr{R}$ is defined as in the second section (Definition 12)), in which they used the notation

$$
\begin{aligned}
& f^{u}=\sup _{t \in \mathbb{T}} f(t), \\
& f^{l}=\inf _{t \in \mathbb{T}} f(t),
\end{aligned}
$$

where $f$ is a bounded function in $\mathbb{T}$.

They first give the definition of the permanence of ecosystem (1), and the definition of the permanence of other ecosystems mentioned in this paper is similar.

Definition 1 (see [13]). System (1) is said to be permanent if there exist $n$-dimensional positive constant vectors $x_{*}, x^{*}, y_{*}$, and $y^{*}$, which are independent on the initial condition, such that, for any solution $\left(x_{i}(t), y_{i}(t)\right)^{\mathbb{T}}, i=1,2, \ldots, n$,

$$
\begin{aligned}
& x_{*} \leq \lim _{t \rightarrow \infty} \inf x_{i}(t) \leq \lim _{t \rightarrow \infty} \sup x_{i}(t) \leq x^{*}, \\
& \qquad i=1,2, \ldots, n, \\
& y_{*} \leq \lim _{t \rightarrow \infty} \inf y_{i}(t) \leq \lim _{t \rightarrow \infty} \sup y_{i}(t) \leq y^{*},
\end{aligned}
$$

$$
i=1,2, \ldots, n \text {. }
$$

Then the main results in [13] can be rewritten as follows.

Theorem 2. Assume that (H1) and (H2) hold, $r_{i}^{l}-\left(d_{i}^{u}+h_{i}^{u}\right) u_{i}^{*}>$ $r_{i}^{l}\left(k_{i}^{-1}+c_{i}^{u}\right)(i=1,2, \ldots, n)$ and $r_{i}^{u}-r_{i}^{l} c_{i}^{l}>0(i=1,2, \ldots, n)$, where $u_{i}^{*}$ can be found in [13]. Then system (1) is permanent.

If $\mathbb{T}=\mathbb{R}$, one refers the readers to $[14,17]$. Chen and Xie [14] considered the following system:

$$
\begin{aligned}
& \frac{d z_{i}(t)}{d t}=z_{i}(t)\left\{r_{i}(t)[1\right. \\
& -\frac{z_{i}(t)}{k_{i}(t)+\sum_{j=1, j \neq i}^{n} e_{i j}(t) \int_{-T_{i j}}^{0} K_{i j}(s) z_{j}(t+s) d s}
\end{aligned}
$$

$$
\begin{aligned}
& \left.-c_{i}(t) z_{i}(t)\right]-d_{i}(t) y_{i}(t)-h_{i}(t) \\
& \left.\cdot \int_{-\tau_{i}}^{0} H_{i}(s) y_{i}(t+s) d s\right\}, \\
& \frac{d y_{i}(t)}{d t}=-a_{i}(t) y_{i}(t)+b_{i}(t) z_{i}(t)+\beta_{i}(t) \int_{-\eta_{i}}^{0} G_{i}(s) \\
& \cdot z_{i}(t+s) d s,
\end{aligned}
$$$$
t \in \mathbb{R}
$$

where $K_{i j}(s), H_{i}(s)$, and $G_{i}(s)$ are all nonnegative continuous functions such that $\int_{-T_{i j}}^{0} K_{i j}(s) d s=1, \int_{-\tau_{i}}^{0} H_{i}(s) d s=1$, and $\int_{-\eta_{i}}^{0} G_{i}(s) z_{i} d s=1, i, j=1,2, \ldots, n$. It is easy to see that the integrations have no influence on the permanence of system (5). They cancelled the additional limitations of [17] and obtained the following.

Theorem 3. Assume that (H1) holds. Then system (5) is permanent.

If $\mathbb{T}=\mathbb{Z}$, one refers the readers to $[15,16]$. Let $N_{i}(t)=$ $\exp \left\{x_{i}(t)\right\}$; they discussed the discrete form of cooperative system (1):

$$
\begin{aligned}
& N_{i}(t+1)=N_{i}(t) \exp \left\{r_{i}(t)[1\right. \\
& -\frac{N_{i}\left(t-\tau_{i i}\right)}{k_{i}(t)+\sum_{j=1, j \neq i}^{n} e_{i j}(t) N_{j}\left(t-\tau_{i j}\right)} \\
& \left.-c_{i}(t) N_{i}\left(t-\tau_{i i}\right)\right]-d_{i}(t) y_{i}(t)-h_{i}(t) y_{i}(t \\
& \left.\left.-\eta_{i}\right)\right\}, \\
& y_{i}(t+1)=\left(1-a_{i}(t)\right) y_{i}(t)+b_{i}(t) N_{i}(t)+\beta_{i}(t) N_{i}(t \\
& \left.-\sigma_{i}\right)
\end{aligned}
$$

$t \in \mathbb{Z}$

In [16], Li and Zhang used the techniques in [11] instead of the comparison theorem in [15] and obtained the following.

Theorem 4. Assume that (H1) holds. Then system (6) is permanent.

Obviously, in Theorem 2, if $\mathbb{T}=\mathbb{R}$ (or $\mathbb{T}=\mathbb{Z}$ ), the conditions are stronger than that in Theorem 3 (or Theorem 4). 
This paper deals with system (1) with the initial condition

$$
x_{i}(\theta) \in \mathbb{R}, y_{i}(\theta) \geq 0 \text { are all } r d \text { - continuous, } \theta \in\left[-\tau, t_{0}\right) \cap \mathbb{T}, y_{i}\left(t_{0}\right)>0, i=1,2, \ldots, n \text {, }
$$

and one assumes that $t-\tau_{i j}, t-\eta_{i}, t-\sigma_{i} \in \mathbb{T}$, for $t \in \mathbb{T}$, which implies that system (1) is valid; $\sup \mathbb{T}=\infty$, which ensures the ultimate boundedness of the solutions; and there exists a positive constant $l$ such that

$$
\mu(t) \leq l
$$

one can see that (8) includes the condition: $\mathbb{T}$ is $p$-periodic (in [13]). In fact, if $\mathbb{T}$ is $p$-periodic, then $\sup \{\mu(t) \mid t \in \mathbb{T}\} \leq p$.

Our aim is to unify Theorems 3 and 4 completely by cancelling the additional limitations of Theorem 2.

For simplicity, one first considers a special case (logistic system) of system (1)

$$
\begin{aligned}
& x^{\Delta}(t)=r(t)\left\{1-\frac{\exp \{x(t)\}}{k(t)}-c(t) y(t)\right\}, \\
& y^{\Delta}(t)=-a(t) y(t)+b(t) \exp \{x(t)\},
\end{aligned}
$$

$$
t \in \mathbb{T}^{k}
$$

with the initial condition

$$
\begin{aligned}
& x\left(t_{0}\right) \in \mathbb{R}, \\
& y\left(t_{0}\right) \geq 0, \\
& t_{0} \in \mathbb{T} ;
\end{aligned}
$$

assume that $1-\mu(t) a(t)>0$ and

(H3) $a, c, k, r, b: \mathbb{T} \rightarrow \mathbb{R}^{+}:=\{x \mid x>0\}$ are all bounded, nonnegative, rd-continuous, and realvalued functions.

Let $z(t)=\exp \{x(t)\} ;$ if $\mathbb{\mathbb { V }}=\mathbb{R}$, then system (9) reduces to the continuous system

$$
\begin{aligned}
& \frac{d z(t)}{d t}=r(t) z(t)\left(1-\frac{z(t)}{k(t)}-c(t) y(t)\right), \\
& \frac{d y(t)}{d t}=-a(t) y(t)+b(t) z(t),
\end{aligned}
$$

$$
t \in \mathbb{R}
$$

In this continuous case, one refers the readers to $[7,19$, 20]. They considered the system with feedback control in periodic case or boundedness case and obtained permanence, stability, and existence of periodic solutions for the system.
Let $N(n)=\exp \{x(n)\}$; if $\mathbb{T}=\mathbb{Z}$, then system (9) is reformulated as

$$
\begin{aligned}
& N(n+1) \\
& \quad=N(n) \exp \left\{r(n)\left(1-\frac{N(n)}{k(n)}-c(n) y(n)\right)\right\}, \\
& \Delta y(n)=-a(n) y(n)+b(n) N(n),
\end{aligned}
$$

$n \in \mathbb{Z}$

this system or its other forms attracted much interest; one refers the readers to $[10-12,21]$. They also considered this system in periodic case or boundedness case and obtained permanence and existence of periodic solutions for this system. Chen [10] discussed the permanence of system (12). Using the comparison theorem, he obtained the following.

Theorem 5. Assume (H3) and

(H4) $c^{u} M_{2}<1$, where $M_{2}=b^{u} M_{1} / a^{l}$ and $M_{1}=$ $\left(k^{u} \exp \left\{r^{u}-1\right\}\right) / r^{u}$

hold. Then system (12) is permanent.

But for system (12) itself, (H4) may not be necessary. Fan and Wang [11] used a method instead of the comparison theorem and obtained the following.

Theorem 6. Assume (H3) holds. Then system (12) is permanent.

One generalizes the method of semicycle [11] to time scales instead of the comparison theorem. By establishing some differential inequalities on time scales, one proves that solutions of systems (1) and (9) are uniformly ultimate bounded. Our result is a unification and extension of Theorems 3 and 4.

The remainder of this paper is arranged as follows. In Section 2, we give some basic properties about time scales and generalize the semicycle concept to time scales. Two differential inequalities on time scales are proved in Section 3. Section 4 is devoted to uniform ultimate boundedness of solutions of systems (1) and (9). An example is presented in Section 5 to support the result. Conclusions are presented in Section 6.

\section{Preliminaries}

First we give some properties about time scales before our main result (see $[1,2])$.

Definition 7 (see $[1,2]$ ). A time scale $\mathbb{T}$ is an arbitrary nonempty closed subset of the real number $\mathbb{R}$. 
Definition 8 (see $[1,2])$. For $t \in \mathbb{T}$ we define the forward jump operator $\sigma: \mathbb{T} \rightarrow \mathbb{T}$ and the backward jump operator $\rho: \mathbb{T} \rightarrow$ T, by

$$
\begin{aligned}
& \sigma(t):=\inf \{s \in \mathbb{T}: s>t\}, \\
& \rho(t):=\sup \{s \in \mathbb{T}: s<t\},
\end{aligned}
$$

respectively.

Throughout the paper we make an assumption that $a \leq b$, $a, b$ are points in $\mathbb{T}$.

Definition 9 (see [2]). Define the interval $[a, b]$ in $\mathbb{~ b y ~}$

$$
[a, b]:=\{t \in \mathbb{T}: a \leq t \leq b\},
$$

and as usual, we assume $t \in \mathbb{T}$, for all $t>T, T \in \mathbb{T}$ is a constant.

We note that $f^{\sigma}(t)=f(\sigma(t))$, for $t \in \mathbb{T}$, where $f^{\sigma}, f$ : $\mathbb{T} \rightarrow \mathbb{R}$.

Lemma 10 (see [2]). If $\mathbb{T}=h \mathbb{Z}=\{h k: k \in \mathbb{Z}\}$, where $h>0$, then

$$
\int_{a}^{b} f(t) \Delta t= \begin{cases}\sum_{k=a / h}^{b / h-1} f(h k) h, & \text { if } a<b, \\ 0, & \text { if } a=b, \\ -\sum_{k=b / h}^{a / h-1} f(h k) h, & \text { if } a>b .\end{cases}
$$

Lemma 11 ((existence of antiderivatives) (see [2])). Every $r d$ continuous function has an antiderivative. In particular if $t_{0} \epsilon$ $\mathbb{T}$, then

$$
F(t):=\int_{t_{0}}^{t} f(\tau) \Delta \tau, \quad \text { for } t \in \mathbb{T}
$$

is an antiderivative of $f$.

Definition 12 (see [2]). We say that a function $p: \mathbb{T} \rightarrow \mathbb{R}$ is regressive provided

$$
1+\mu(t) p(t) \neq 0 \quad \text { for all } t \in \mathbb{T}^{k}
$$

holds, where throughout the paper

$$
\mathbb{T}^{k}= \begin{cases}\mathbb{T} \backslash(\rho(\sup \mathbb{T}), \sup \mathbb{\mathbb { T }}], & \text { if } \sup \mathbb{T}<\infty, \\ \mathbb{T}, & \text { if } \sup \mathbb{T}=\infty .\end{cases}
$$

The set of all regressive and rd-continuous functions $f: \mathbb{T} \rightarrow$ $\mathbb{R}$ will be denoted in this paper by

$$
\mathscr{R}=\mathscr{R}(\mathbb{T})=\mathscr{R}(\mathbb{T}, \mathbb{R}) .
$$

Definition 13 (see [2]). If $p \in \mathscr{R}$, we define the exponential function by

$$
e_{p}(t, s)=\exp \left\{\int_{s}^{t} \xi_{\mu(\tau)}(p(\tau)) \Delta \tau\right\}, \quad \text { for } s, t \in \mathbb{T},
$$

where the cylinder transformation $\xi_{h}(z)=(1 / h) \log (1+z h)$, for $h>0$.

For $h=0$, we define $\xi_{0}(z)=z$. In this case, $\mathbb{T}=\mathbb{R}, \mu(t)=$ 0 , and $e_{p}(t, s)=\exp \left\{\int_{s}^{t} p(\tau) \Delta \tau\right\}$.

When $\mathbb{T}=h \mathbb{Z}=\{h k: k \in \mathbb{Z}\}$, we know that $\mu(h k)=$ $h(k+1)-h k=h>0$; then for $s<t$,

$$
\begin{aligned}
e_{p}(t, s) & =\exp \left\{\int_{s}^{t} \frac{1}{h} \log _{e}(1-h a(\tau)) \Delta \tau\right\} \\
& =\exp \left\{\sum_{k=s / h}^{t / h-1} \log _{e}(1-h a(h k))\right\} \\
& =\prod_{k=s / h}^{t / h-1}\{1-h a(h k)\} .
\end{aligned}
$$

Definition 14 (see [2]). If $p \in \mathscr{R}$, the function $\ominus(p)$ is defined by

$$
\ominus(p)(t)=-\frac{p(t)}{1+\mu(t) p(t)}, \quad \text { for } t \in \mathbb{T}^{k}
$$

Lemma 15 (see [2]). Suppose $p \in \mathscr{R}$; then
(1) $\ominus(\ominus(p))=p$;
(2) $e_{p}(\sigma(t), s)=(1+\mu(t) p(t)) e_{p}(t, s)$;
(3) $1 / e_{p}(t, s)=e_{\ominus p}(t, s)$;
(4) $\left(1 / e_{p}(\cdot, s)\right)^{\Delta}=-p(\cdot) / e_{p}^{\sigma}(\cdot, s)$;
(5) $\left(e_{p}(\cdot, s)\right)^{\Delta}=p(\cdot) e_{p}(\cdot, s)$.

Similar to the definition of semicycle in discrete case [22], one gives the following.

Definition 16. Let $m$ be a constant and : $\mathbb{T} \rightarrow \mathbb{R}$; a positive semicycle relative to $m$ of $f$ is defined as follows: it consists of a "string" of terms

$$
\{f(t), t \in[s, t], s, t \in \mathbb{T}\}
$$

all greater than or equal to $m$; and a negative semicycle relative to $m$ of $f$ is defined as follows: it is a "string" of terms

$$
\{f(t), t \in[p, q], p, q \in \mathbb{T}\}
$$

all less than or equal to $m$.

\section{Differential Inequalities}

By using the semicycle concept and the comparison theorem, we give some differential inequalities as follows.

Lemma 17. Assume that $A, B>0$ and $x_{1}(0)=x_{0}>0$, and further suppose that $x_{1}(t)$ satisfies the following:

(i)

$$
x_{1}^{\Delta}(t) \leq B-A \exp \left\{x_{1}(t)\right\}, \text { for } t \geq 0 ;
$$


then

$$
\lim _{t \rightarrow \infty} \sup x_{1}(t) \leq B l+\ln \frac{B}{A} .
$$

(ii)

$$
x_{1}^{\Delta}(t) \geq B-A \exp \left\{x_{1}(t)\right\}, \quad \text { for } t \geq 0,
$$

and there exists a constant $M$, such that $\lim _{t \rightarrow \infty} \sup x_{1}(t)<M$; then

$$
\lim _{t \rightarrow \infty} \inf x_{1}(t) \geq(B-A \exp \{M\}) l+\ln \frac{B}{A} .
$$

Proof. (i) According to the oscillating property of $x_{1}(t)$, we divided the proof into two cases. Firstly we assume that $x_{1}(t)$ does not oscillate ultimately about $\ln B / A$. If there exists a constant $T_{1}>0$, such that $x_{1}(t) \leq \ln B / A$, for $t>T_{1}$, then

$$
x_{1}(t) \leq \ln \frac{B}{A} \leq B l+\ln \frac{B}{A} ;
$$

thus (26) holds true. If there exists a constant $T_{1}>0$, such that $x_{1}(t)>\ln B / A$, for $t>T_{1}$, then $x_{1}^{\Delta}(t)<0$, which means that $x_{1}(t)$ is eventually decreasing; therefore, $\lim _{t \rightarrow \infty} x_{1}(t)$ exists. Then (25) yields $\lim _{t \rightarrow \infty} x_{1}(t)=\ln B / A$, which leads to

$$
\lim _{t \rightarrow \infty} \sup x_{1}(t)=\ln \frac{B}{A} \leq B l+\ln \frac{B}{A} .
$$

Finally, we assume that $x_{1}(t)$ oscillates about $\ln B / A$, by (25), we know that $x_{1}(t) \geq \ln B / A$ implies $x_{1}^{\Delta}(t) \leq 0$. Thus, by semicycle concept, we assume that $x_{1}(t) \geq \ln B / A$, for $t \epsilon$ $\left[s_{\alpha}, t_{\alpha}\right], \alpha \in \mathscr{L}$, where $\mathscr{L}$ is an index set, and the interval $\left[s_{\alpha}, t_{\alpha}\right]$ satisfies the following:

(a) For any $\alpha, \beta \in \mathscr{L}$, if $\alpha \neq \beta,\left[s_{\alpha}, t_{\alpha}\right] \cap\left[s_{\beta}, t_{\beta}\right]=\varnothing$.

(b) If $s_{\alpha}$ is left-scattered, then $x_{1}\left(\rho\left(s_{\alpha}\right)\right)<\ln B / A$.

(c) If $s_{\alpha}$ is left-dense, then there exists a hollow left neighbourhood $\stackrel{o}{U}_{-}\left(s_{\alpha}\right)$ of $s_{\alpha}$ such that $x_{1}(t)<\ln B / A$, for $t \in \stackrel{o}{U}_{-}\left(s_{\alpha}\right)$.

(d) If $t_{\alpha}$ is right-scattered, then $x_{1}\left(\sigma\left(t_{\alpha}\right)\right)<\ln B / A$.

(e) If $t_{\alpha}$ is right-dense, then there exists a hollow right neighbourhood $\stackrel{o}{U}_{+}\left(t_{\alpha}\right)$ of $t_{\alpha}$ such that $x_{1}(t)<\ln B / A$, for $t \in \stackrel{o}{U}_{+}\left(t_{\alpha}\right)$.

Notice that $\lim _{t \rightarrow \infty} \sup x_{1}(t)=\lim _{\alpha \rightarrow \infty} \sup x_{1}\left(s_{\alpha}\right)$. If $s_{\alpha}$ is left-scattered, by integrating (25) over the set $\left[\rho\left(s_{\alpha}\right), s_{\alpha}\right]$, we have

$$
\begin{aligned}
x_{1}\left(s_{\alpha}\right)-x_{1}\left(\rho\left(s_{\alpha}\right)\right) & \leq \int_{\rho\left(s_{\alpha}\right)}^{s_{\alpha}}\left(B-A \exp \left\{x_{1}(t)\right\}\right) \Delta t \\
& \leq \int_{\rho\left(s_{\alpha}\right)}^{s_{\alpha}} B \Delta t,
\end{aligned}
$$

by (b), it follows that

$$
x_{1}\left(s_{\alpha}\right) \leq \ln \frac{B}{A}+\int_{\rho\left(s_{\alpha}\right)}^{s_{\alpha}} B \Delta t,
$$

and from (8), we know $s_{\alpha}-\rho\left(s_{\alpha}\right) \leq l$; then $x_{1}\left(s_{\alpha}\right) \leq B l+$ $\ln B / A$.

If $s_{\alpha}$ is left-dense, we choose $t_{1} \in \stackrel{o}{U_{-}}\left(s_{\alpha}\right)$, such that $s_{\alpha}-$ $t_{1} \leq l$. By integrating (25) over the set $\left[t_{1}, s_{\alpha}\right]$, we have

$$
\begin{aligned}
x_{1}\left(s_{\alpha}\right)-x_{1}\left(t_{1}\right) & \leq \int_{t_{1}}^{s_{\alpha}}\left(B-A \exp \left\{x_{1}(t)\right\}\right) \Delta t \\
& \leq \int_{t_{1}}^{s_{\alpha}} B \Delta t,
\end{aligned}
$$

notice that $x_{1}\left(t_{1}\right)<\ln B / A$, and it follows that

$$
x_{1}\left(s_{\alpha}\right) \leq \ln \frac{B}{A}+\int_{t_{1}}^{s_{\alpha}} B \Delta t \leq B l+\ln \frac{B}{A} .
$$

Then

$$
\lim _{t \rightarrow \infty} \sup x_{1}(t)=\lim _{\alpha \rightarrow \infty} \sup x_{1}\left(s_{\alpha}\right) \leq B l+\ln \frac{B}{A},
$$

which completes the proof of (i).

(ii) The proof is similar to (i) of Lemma 17.

Lemma 18. Assume that $C(t), D(t)>0$ are bounded and $r d-$ continuous functions, $1-C(t) \mu(t)>0$, and $C^{l}>0$; further suppose that $y_{1}(t)$ satisfies the following:

(iii)

$$
y_{1}^{\Delta}(t)+C(t) y_{1}(t) \leq D(t), \quad \text { for } t \geq T_{0} ;
$$

then there exists a constant $T_{2}>T_{0}$, such that, for $t>T_{2}$, one has

$$
y_{1}(t) \leq y_{1}\left(T_{2}\right) e_{(-C)}\left(t, T_{2}\right)+\frac{D(t)}{C^{l}} .
$$

Particularly, if $D(t)$ is bounded above ultimately with respect to $K_{1}$, then

$$
\limsup _{t \rightarrow \infty} y_{1}(t) \leq \frac{K_{1}}{C^{l}} .
$$

(iv)

$$
y_{1}^{\Delta}(t)+C(t) y_{1}(t) \geq D(t), \quad \text { for } t \geq T_{0} ;
$$

there exists a constant $T_{3}>T_{0}$, such that for, $t>T_{3}$, one has

$$
y_{1}(t) \geq\left(y_{1}\left(T_{3}\right)-\frac{D\left(T_{3}\right)}{C^{u}}\right) e_{(-C)}\left(t, T_{3}\right)+\frac{D\left(T_{3}\right)}{C^{u}} .
$$

Particularly, if $D(t)$ is bounded below ultimately with respect to $k_{1}$, then

$$
\liminf _{t \rightarrow \infty} y_{1}(t) \geq \frac{k_{1}}{C^{u}} .
$$

Proof. (iii) From $1-C(t) \mu(t)>0$, we have $-C \in \mathscr{R}$. Note that $0 \leq e_{(-C)}^{\sigma}\left(t, T_{0}\right) \leq e_{(-C)}\left(t, T_{0}\right) \rightarrow 0$, when $t \rightarrow \infty$. Hence, 
there exists a $T_{2}>T_{0}$, such that $e_{(-C)}^{\sigma}\left(t, T_{2}\right) \leq 1$, as $t>T_{2}$. We use the product rule to calculate

$$
\begin{aligned}
{\left[y_{1} e_{\ominus(-C)}\left(t, T_{2}\right)\right]^{\Delta}(t) } \\
=y_{1}^{\Delta}(t) e_{\ominus(-C)}^{\sigma}\left(t, T_{2}\right) \\
\quad+y_{1}(t)(\ominus(-C))(t) e_{\ominus(-C)}\left(t, T_{2}\right) \\
=y_{1}^{\Delta}(t) e_{\ominus(-C)}\left(\sigma(t), T_{2}\right) \\
\quad+y_{1}(t) \frac{(\ominus(-C))(t)}{1+(\sigma(t)-t)(\ominus(-C))(t)} e_{\ominus(-C)}^{\sigma}\left(t, T_{2}\right) \\
=\left[y_{1}^{\Delta}(t)-(\ominus(\ominus(-C)))(t) y_{1}(t)\right] e_{\ominus(-C)}^{\sigma}\left(t, T_{2}\right) \\
=\left[y_{1}^{\Delta}(t)+C(t) y_{1}(t)\right] e_{\ominus(-C)}^{\sigma}\left(t, T_{2}\right),
\end{aligned}
$$

noticing that $D(t)$ is rd-continuous, from Lemma 11,

$$
D(t)=\int_{T_{2}}^{t} d(\tau) \Delta \tau, \quad \text { for } t \in \mathbb{T}
$$

and by integrating both sides over the set $\left[T_{2}, t\right]$, we have

$$
\begin{aligned}
y_{1}(t) e_{\ominus(-C)}\left(t, T_{2}\right)-y_{1}\left(T_{2}\right) \\
=\int_{T_{2}}^{t}\left[y_{1}^{\Delta}(\tau)+C(\tau) y_{1}(\tau)\right] e_{\ominus(-C)}^{\sigma}\left(\tau, T_{2}\right) \Delta \tau \\
\leq \int_{T_{2}}^{t} D(\tau) e_{\ominus(-C)}^{\sigma}\left(\tau, T_{2}\right) \Delta \tau \\
=\int_{T_{2}}^{t} D(\tau) \frac{1}{e_{(-C)}^{\sigma}\left(\tau, T_{2}\right)} \Delta \tau \\
=\int_{T_{2}}^{t} \frac{D(\tau)}{C(\tau)} \frac{C(\tau)}{e_{(-C)}^{\sigma}\left(\tau, T_{2}\right)} \Delta \tau \\
\leq \frac{1}{C^{l}} \int_{T_{2}}^{t} \frac{D(\tau) C(\tau)}{e_{(-C)}^{\sigma}\left(\tau, T_{2}\right)} \Delta \tau \\
\leq \frac{1}{C^{l}}\left[\left.\frac{D(t)}{e_{(-C)}\left(t, T_{2}\right)}\right|_{T_{2}} ^{t}-\int_{T_{2}}^{t} \frac{d(\tau)}{e_{(-C)}^{\sigma}\left(\tau, T_{2}\right)} \Delta \tau\right] \\
=\frac{1}{C^{l}}\left[\frac{D(t)}{e_{(-C)}\left(t, T_{2}\right)}-D\left(T_{2}\right)-\int_{T_{2}}^{t} \frac{d(\tau)}{e_{(-C)}^{\sigma}\left(\tau, T_{2}\right)} \Delta \tau\right] \\
\leq \frac{1}{C^{l}}\left(\frac{D(t)}{e_{(-C)}\left(t, T_{2}\right)}-D\left(T_{2}\right)-\int_{T_{2}}^{t} d(\tau) \Delta \tau\right) \\
=\frac{1}{C^{l}}\left(\frac{D(t)}{e_{(-C)}\left(t, T_{2}\right)}-D(t)\right) \leq \frac{1}{C^{l}} \frac{D(t)}{e_{(-C)}\left(t, T_{2}\right)} ;
\end{aligned}
$$

then

$$
\begin{aligned}
y_{1}(t) \leq & y_{1}\left(T_{2}\right) e_{(-C)}\left(t, T_{2}\right) \\
& +e_{(-C)}\left(t, T_{2}\right) \frac{1}{C^{l}}\left(\frac{D(t)}{e_{(-C)}\left(t, T_{2}\right)}\right) \\
= & y_{1}\left(T_{2}\right) e_{(-C)}\left(t, T_{2}\right)+\frac{D(t)}{C^{l}} .
\end{aligned}
$$

Thus

$$
\limsup _{t \rightarrow \infty} y_{1}(t) \leq \frac{K_{1}}{C^{l}} .
$$

(iv) From (iii) of Lemma 18, we know that there exists a constant $T_{3}>T_{0}$, such that $D\left(T_{3}\right)=k_{1}$ and $D(t)=$ $\int_{T_{3}}^{t} d(\tau) \Delta \tau$; then for $t>T_{3}$,

$$
\begin{aligned}
& y_{1}(t) e_{\Theta(-C)}\left(t, T_{3}\right)-y_{1}\left(T_{3}\right) \\
& \quad=\int_{T_{3}}^{t}\left[y_{1}^{\Delta}(\tau)+C(\tau) y_{1}(\tau)\right] e_{\Theta(-C)}^{\sigma}\left(\tau, T_{3}\right) \Delta \tau ;
\end{aligned}
$$

then

$$
y_{1}(t) \geq y_{1}\left(T_{3}\right) e_{(-C)}\left(t, T_{3}\right)+e_{(-C)}\left(t, T_{3}\right)
$$

$$
\cdot \int_{T_{3}}^{t} D(\tau) e_{\ominus(-C)}^{\sigma}\left(\tau, T_{3}\right) \Delta \tau=y_{1}\left(T_{3}\right)
$$$$
\cdot e_{(-C)}\left(t, T_{3}\right)+e_{(-C)}\left(t, T_{3}\right)
$$$$
\cdot \int_{T_{3}}^{t} \frac{D(\tau)}{C(\tau)} \frac{C(\tau)}{e_{(-C)}^{\sigma}\left(\tau, T_{3}\right)} \Delta \tau \geq y_{1}\left(T_{3}\right) e_{(-C)}\left(t, T_{3}\right)
$$$$
+e_{(-C)}\left(t, T_{3}\right) \frac{1}{C^{u}} \int_{T_{3}}^{t} \frac{D(\tau) C(\tau)}{e_{(-C)}^{\sigma}\left(\tau, T_{3}\right)} \Delta \tau=y_{1}\left(T_{3}\right)
$$$$
\cdot e_{(-C)}\left(t, T_{3}\right)+e_{(-C)}\left(t, T_{3}\right) \frac{1}{C^{u}}\left[\left.\frac{D(t)}{e_{(-C)}\left(t, T_{3}\right)}\right|_{T_{3}} ^{t}\right.
$$$$
\left.-\int_{T_{3}}^{t} \frac{d(\tau)}{e_{(-C)}^{\sigma}\left(\tau, T_{3}\right)} \Delta \tau\right]=y_{1}\left(T_{3}\right) e_{(-C)}\left(t, T_{3}\right)
$$$$
+e_{(-C)}\left(t, T_{3}\right) \frac{1}{C^{u}}\left[\frac{D(t)}{e_{(-C)}\left(t, T_{3}\right)}-D\left(T_{3}\right)\right.
$$$$
\left.-\int_{T_{3}}^{t} \frac{d(\tau)}{e_{(-C)}^{\sigma}\left(\tau, T_{3}\right)} \Delta \tau\right] \geq y_{1}\left(T_{3}\right) e_{(-C)}\left(t, T_{3}\right)
$$$$
+\frac{D(t)}{C^{u}}-\frac{e_{(-C)}\left(t, T_{3}\right)}{C^{u}} D\left(T_{3}\right)-\frac{1}{C^{u}}
$$$$
\cdot \int_{T_{3}}^{t} \frac{e_{(-C)}\left(t, T_{3}\right)}{e_{(-C)}^{\sigma}\left(\tau, T_{3}\right)} d(\tau) \Delta \tau \geq y_{1}\left(T_{3}\right) e_{(-C)}\left(t, T_{3}\right)
$$$$
+\frac{D(t)}{C^{u}}-\frac{e_{(-C)}\left(t, T_{3}\right)}{C^{u}} D\left(T_{3}\right)-\frac{1}{C^{u}} \int_{T_{3}}^{t} d(\tau) \Delta \tau
$$ 


$$
\begin{aligned}
& \geq y_{1}\left(T_{3}\right) e_{(-C)}\left(t, T_{3}\right)+\frac{D(t)}{C^{u}}+\left(\frac{1}{C^{u}}\right. \\
& \left.-\frac{e_{(-C)}\left(t, T_{3}\right)}{C^{u}}\right) D\left(T_{3}\right)-\frac{D(t)}{C^{u}} \geq\left(y_{1}\left(T_{3}\right)\right. \\
& \left.-\frac{D\left(T_{3}\right)}{C^{u}}\right) e_{(-C)}\left(t, T_{3}\right)+\frac{D\left(T_{3}\right)}{C^{u}} .
\end{aligned}
$$

Thus,

$$
\liminf _{t \rightarrow \infty} y_{1}(t) \geq \frac{k_{1}}{C^{u}} .
$$

Then the proof of Lemma 18 is completed.

\section{Applications}

Before giving our main result, we list the definition of uniform ultimate boundedness.

Definition 19. The solution $\left(x_{1}(t), x_{2}(t)\right)^{\mathbb{T}}$ of system (9) is said to be uniformly ultimate bounded if there exist two constants $\lambda_{1}$ and $\lambda_{2}$ such that, for any initial condition $\left(x_{1}(0), x_{2}(0)\right)^{\mathbb{T}} \in$ $\mathbb{R}^{2}$,

$$
\lambda_{1} \leq \liminf _{t \rightarrow \infty} x_{i}(t) \leq \lim _{t \rightarrow \infty} \sup x_{i}(t) \leq \lambda_{2}, \quad i=1,2,
$$

where $\lambda_{1}$ and $\lambda_{2}$ are independent on $\left(x_{1}(0), x_{2}(0)\right)^{\mathbb{T}}$.

The definition of the uniform ultimate boundedness of solutions of $\mathrm{N}$-species system is similar.

Then we give the applications of these differential inequalities in this section.

Theorem 20. Assume that (H3) holds, $a^{l}, k^{l}, r^{l}>0$. Then the solutions for system (9) are uniformly ultimate bounded.

Proof. From $1-\mu(t) a(t)>0$, we have $-a \in \mathscr{R}$. From (9) and $y\left(t_{0}\right) \geq 0$, we can see

$$
\begin{aligned}
& y(t) \\
& =y\left(t_{0}\right) e_{(-a)}\left(t, t_{0}\right) \\
& \quad+e_{(-a)}\left(t, t_{0}\right) \int_{t_{0}}^{t} b(\tau) \exp \{x(\tau)\} e_{\ominus(-a)}^{\sigma}\left(\tau, t_{0}\right) \Delta \tau
\end{aligned}
$$

$$
\geq 0
$$

then

$$
\begin{aligned}
x^{\Delta}(t) & \leq r(t)-\frac{r(t)}{k(t)} \exp \{x(t)\} \\
& \leq r^{u}-\frac{r^{l}}{k^{u}} \exp \{x(t)\} ;
\end{aligned}
$$

from Lemma 17, we have

$$
\lim _{t \rightarrow \infty} \sup x(t) \leq r^{u} l+\ln \frac{r^{u} k^{u}}{r^{l}}:=M_{1} \text {. }
$$

From (9) and (53), there exists a constant $T_{2}>t_{0}$, such that, for $t>T_{2}$,

$$
y^{\Delta}(t)+a(t) y(t)=b(t) \exp \{x(t)\} \leq b^{u} \exp \left\{M_{1}\right\} ;
$$

from Lemma 18, we have

$$
\lim _{t \rightarrow \infty} \sup y(t) \leq \frac{b^{u} \exp \left\{M_{1}\right\}}{a^{l}}:=M_{2} .
$$

From (9) and Lemma 18,

$$
y^{\Delta}(t)+a(t) y(t)=b(t) \exp \{x(t)\} \leq b^{u} \exp \{x(t)\} ;
$$

then

$$
y(t) \leq y\left(T_{2}\right) e_{(-a)}\left(t, T_{2}\right)+\frac{b^{u} \exp \{x(t)\}}{a^{l}} .
$$

Note that $0 \leq y\left(T_{2}\right) e_{(-a)}\left(t, T_{2}\right) \rightarrow 0$, when $t \rightarrow \infty$. Hence, there exists $T_{4}$, such that $c^{u} y\left(T_{2}\right) e_{(-a)}\left(T_{4}, T_{2}\right) \leq 1 / 2$, for $t>$ $T_{4}$. Then we get

$$
y(t) \leq y\left(T_{2}\right) e_{(-a)}\left(T_{4}, T_{2}\right)+\frac{b^{u} \exp \{x(t)\}}{a^{l}},
$$

for $t>T_{4}$.

Note that $y\left(T_{2}\right) e_{(-a)}\left(T_{4}, T_{2}\right):=K$, from (9) and (58), when $t>T_{4}$,

$$
\begin{aligned}
& x^{\Delta}(t) \geq r(t)\left\{1-\frac{1}{k(t)} \exp \{x(t)\}\right. \\
& \left.-c(t)\left[K+\frac{b^{u} \exp \{x(t)\}}{a^{l}}\right]\right\}=r(t)(1 \\
& \left.-\frac{1}{k(t)} \exp \{x(t)\}\right)-r(t) c(t)\{K \\
& \left.+\frac{b^{u} \exp \{x(t)\}}{a^{l}}\right\}=r(t)\{1-c(t) K\}-\left(\frac{r(t)}{k(t)}\right. \\
& \left.+\frac{r(t) c(t) b^{u}}{a^{l}}\right) \exp \{x(t)\} \geq r^{l}\left\{1-c^{u} K\right\}-\left(\frac{r^{u}}{k^{l}}\right. \\
& \left.+\frac{r^{u} c^{u} b^{u}}{a^{l}}\right) \exp \{x(t)\} ;
\end{aligned}
$$

noticing that $r^{l}-r^{l} c^{u} K \geq r^{l} / 2>0$, from Lemma 17, we have

$$
\begin{aligned}
& \lim _{t \rightarrow \infty} \inf x(t) \\
& \geq\left[r^{l}\left(1-c^{u} K\right)-\left(\frac{r^{u}}{k^{l}}+\frac{r^{u} c^{u} b^{u}}{a^{l}}\right) \exp \left\{M_{1}\right\}\right] l \\
& \quad+\ln \frac{a^{l} k^{l} r^{l}\left(1-c^{u} K\right)}{a^{l} r^{u}+k^{l} r^{u} c^{u} b^{u}}:=m_{1} .
\end{aligned}
$$

From (9) and (60), there exists a constant $T_{5}>T_{4}$, such that, for $t>T_{5}$,

$$
y^{\Delta}(t)+a(t) y(t)=b(t) \exp \{x(t)\} \geq b^{l} \exp \left\{m_{1}\right\}
$$


from Lemma 18, we have

$$
\lim _{t \rightarrow \infty} \inf y(t) \geq \frac{b^{l} \exp \left\{m_{1}\right\}}{a^{u}}:=m_{2} .
$$

From (53), (55), (60), and (62), we obtain

$$
\begin{aligned}
& m_{1} \leq \liminf _{t \rightarrow \infty} x(t) \leq \lim _{t \rightarrow \infty} \sup x(t) \leq M_{1}, \\
& m_{2} \leq \liminf _{t \rightarrow \infty} y(t) \leq \lim _{t \rightarrow \infty} \sup y(t) \leq M_{2} ;
\end{aligned}
$$

then the solutions for system (9) are uniformly ultimately bounded.

This completes the proof of Theorem 20 .

\section{Theorem 21. Assume the assumption}

(H5) $r_{i}(t), a_{i}(t), b_{i}(t), c_{i}(t), d_{i}(t), e_{i j}(t), h_{i}(t), k_{i}(t)$, and $\beta_{i}(t)(i, j=1,2, \ldots, n)$ are all bounded, nonnegative, rd-continuous, and real-valued functions; $\tau_{i j}, \eta_{i}, \sigma_{i}(i, j=1,2, \ldots, n)$ are positive constants. $\tau=$ $\max \left\{\max _{1 \leq i, j \leq n} \tau_{i j}, \max _{1 \leq i \leq n} \sigma_{i}, \max _{1 \leq i \leq n} \eta_{i}\right\}>0$

holds; $r_{i}^{l} c_{i}^{l}, a_{i}^{l}, k_{i}^{l}>0$. Then the solutions of system (1) are uniformly ultimate bounded.

Proof. By similar analysis as that in Theorem 20 and the initial condition (7), we have $y_{i}(t) \geq 0$. From (1), we can see $x_{i}^{\Delta}(t) \leq$ $r_{i}(t) \leq r_{i}^{u}$, by integrating both sides over the set $\left[t-\tau_{i i}, t\right]$,

$$
\begin{aligned}
x_{i}(t)-x_{i}\left(t-\tau_{i i}\right) & =\int_{t-\tau_{i i}}^{t} x_{i}^{\Delta}(t) \Delta t \leq \int_{t-\tau_{i i}}^{t} r_{i}^{u} \Delta t \\
& =\tau_{i i} r_{i}^{u},
\end{aligned}
$$

so $\exp \left\{x_{i}\left(t-\tau_{i i}\right)\right\} \geq \exp \left\{x_{i}(t)-\tau_{i i} r_{i}^{u}\right\}$; then the first equation of (1) implies

$$
\begin{aligned}
x_{i}^{\Delta}(t) & \leq r_{i}(t)-r_{i}(t) c_{i}(t) \exp \left\{x_{i}\left(t-\tau_{i i}\right)\right\} \\
& \leq r_{i}^{u}-r_{i}^{l} c_{i}^{l} \exp \left\{x_{i}\left(t-\tau_{i i}\right)\right\} \\
& \leq r_{i}^{u}-\frac{r_{i}^{l} c_{i}^{l}}{\exp \left\{\tau_{i i} r_{i}^{u}\right\}} \exp \left\{x_{i}(t)\right\} ;
\end{aligned}
$$

from Lemma 17, we have

$$
\lim _{t \rightarrow \infty} \sup x_{i}(t) \leq r_{i}^{u} l+\tau_{i i} r_{i}^{u} \ln \frac{r_{i}^{u}}{r_{i}^{l} c_{i}^{l}}:=M_{i 1} .
$$

From (1) and (66), there exists a constant $T_{2}>t_{0}$, such that, for $t>T_{2}$,

$$
\begin{aligned}
y_{i}^{\Delta}(t)+a_{i}(t) y_{i}(t)= & b_{i}(t) \exp \left\{x_{i}(t)\right\} \\
& +\beta_{i}(t) \exp \left\{x_{i}\left(t-\sigma_{i}\right)\right\} \\
\leq & \left(b_{i}^{u}+\beta_{i}^{u}\right) \exp \left\{M_{\mathrm{i} 1}\right\} ;
\end{aligned}
$$

from Lemma 18, we have

$$
\limsup _{t \rightarrow \infty} y_{i}(t) \leq \frac{\left(b_{i}^{u}+\beta_{i}^{u}\right) \exp \left\{M_{i 1}\right\}}{a_{i}^{l}}:=M_{i 2} .
$$

From (1), (66), and (68), we have

$$
\begin{aligned}
x_{i}^{\Delta}(t) \geq & r_{i}^{l}-r_{i}^{u} \frac{\exp \left\{M_{i 1}\right\}}{k_{i}^{l}}-r_{i}^{u} c_{i}^{u} \exp \left\{M_{i 1}\right\} \\
& -\left(d_{i}^{u}+h_{i}^{u}\right) M_{i 2}:=k_{i 2} ;
\end{aligned}
$$

by integrating both sides over the set $\left[t-\sigma_{i}, t\right]$, we have $x_{i}(t)-$ $x_{i}\left(t-\sigma_{i}\right) \geq \sigma_{i} k_{i 2}$; thus from (1) and Lemma 18,

$$
\begin{aligned}
y_{i}^{\Delta} & (t)+a_{i}(t) y_{i}(t) \\
& =b_{i}(t) \exp \left\{x_{i}(t)\right\}+\beta_{i}(t) \exp \left\{x_{i}\left(t-\sigma_{i}\right)\right\} \\
& \leq\left(b_{i}^{u}+\frac{\beta_{i}^{u}}{\exp \left\{\sigma_{i} k_{i 2}\right\}}\right) \exp \left\{x_{i}(t)\right\} ;
\end{aligned}
$$

then

$$
\begin{aligned}
y_{i}(t) \leq & y_{i}\left(T_{2}\right) e_{\left(-a_{i}\right)}\left(t, T_{2}\right) \\
& +\left(b_{i}^{u}+\frac{\beta_{i}^{u}}{\exp \left\{\sigma_{i} k_{i 2}\right\}}\right) \exp \left\{x_{i}(t)\right\} .
\end{aligned}
$$

Note that $0 \leq y_{i}\left(T_{2}\right) e_{\left(-a_{i}\right)}\left(t, T_{2}\right) \rightarrow 0$, when $t \rightarrow \infty$. Hence, there exists $T_{6}$ such that

$$
\left(d_{i}^{u}+h_{i}^{u}\right) y_{i}\left(T_{2}\right) e_{\left(-a_{i}\right)}\left(T_{6}, T_{2}\right) \leq \frac{r_{i}^{l}}{2},
$$

for $t>T_{6}$. Then we get

$$
\begin{aligned}
y_{i}(t) \leq & y_{i}\left(T_{2}\right) e_{\left(-a_{i}\right)}\left(T_{6}, T_{2}\right) \\
& +\left(b_{i}^{u}+\frac{\beta_{i}^{u}}{\exp \left\{\sigma_{i} k_{i 2}\right\}}\right) \exp \left\{x_{i}(t)\right\},
\end{aligned}
$$

for $t>T_{6}$.

Let $K_{i}=y_{i}\left(T_{2}\right) e_{\left(-a_{i}\right)}\left(T_{6}, T_{2}\right)$ and $k_{i 3}=b_{i}^{u}+\beta_{i}^{u} / \exp \left\{\sigma_{i} k_{2}\right\}$; noticing that $x_{i}(t)-x_{i}\left(t-\eta_{i}\right) \geq \eta_{i} k_{i 2}$ and $x_{i}(t)-x_{i}\left(t-\tau_{i i}\right) \geq$ $\tau_{i i} k_{i 2}$, from (1) and (73), when $t>T_{6}$,

$$
\begin{aligned}
& x_{i}^{\Delta}(t)=r_{i}(t)\{1 \\
& -\frac{\exp \left\{x_{i}\left(t-\tau_{i i}\right)\right\}}{k_{i}(t)+\sum_{j=1, j \neq i}^{n} e_{i j}(t) \exp \left\{x_{j}\left(t-\tau_{i j}\right)\right\}} \\
& \left.-c_{i}(t) \exp \left\{x_{i}\left(t-\tau_{i i}\right)\right\}\right\}-d_{i}(t) y_{i}(t)-h_{i}(t) y_{i}(t \\
& \left.-\eta_{i}\right) \geq r_{i}(t)\left\{1-\frac{\exp \left\{x_{i}\left(t-\tau_{i i}\right)\right\}}{k_{i}(t)}\right. \\
& \left.-c_{i}(t) \exp \left\{x_{i}\left(t-\tau_{i i}\right)\right\}\right\}-d_{i}(t)\left(K_{i}\right.
\end{aligned}
$$




$$
\begin{aligned}
& \left.+k_{i 3} \exp \left\{x_{i}(t)\right\}\right)-h_{i}(t)\left(K_{i}\right. \\
& \left.+k_{i 3} \exp \left\{x_{i}\left(t-\eta_{i}\right)\right\}\right) \geq r_{i}(t)\{1 \\
& \left.-\frac{\exp \left\{x_{i}\left(t-\tau_{i i}\right)\right\}}{k_{i}(t)}-c_{i}(t) \exp \left\{x_{i}\left(t-\tau_{i i}\right)\right\}\right\} \\
& -d_{i}(t)\left(K_{i}+k_{i 3} \exp \left\{x_{i}(t)\right\}\right)-h_{i}(t)\left(K_{i}\right. \\
& \left.+\frac{k_{i 3}}{\exp \left\{\eta_{i} k_{i 2}\right\}} \exp \left\{x_{i}(t)\right\}\right) \geq r_{i}^{l}-\left(d_{i}^{u}+h_{i}^{u}\right) K_{i} \\
& -\left(d_{i}^{u}+h_{i}^{u}\right)\left(k_{i 3}+\frac{k_{i 3}}{\exp \left\{\eta_{i} k_{i 2}\right\}}\right) \exp \left\{x_{i}(t)\right\} \\
& -\frac{r_{i}^{u}\left(k_{i}^{-l}+c_{i}^{u}\right)}{\exp \left\{\tau_{i i} k_{i 2}\right\}} \exp \left\{x_{i}(t)\right\} \geq r_{i}^{l}-\left(d_{i}^{u}+h_{i}^{u}\right) K_{i} \\
& -\left[\left(d_{i}^{u}+h_{i}^{u}\right)\left(k_{i 3}+\frac{k_{i 3}}{\exp \left\{\eta_{i} k_{i 2}\right\}}\right)\right. \\
& \left.+\frac{r_{i}^{u}\left(k_{i}^{-l}+c_{i}^{u}\right)}{\exp \left\{\tau_{i i} k_{i 2}\right\}}\right] \exp \left\{x_{i}(t)\right\},
\end{aligned}
$$

noticing that $r_{i}^{l}-\left(d_{i}^{u}+h_{i}^{u}\right) K_{i} \geq r_{i}^{l} / 2>0$, denote $\left(d_{i}^{u}+\right.$ $\left.h_{i}^{u}\right)\left(k_{i 3}+k_{i 3} / \exp \left\{\eta_{i} k_{i 2}\right\}\right)+r_{i}^{u}\left(k_{i}^{-l}+c_{i}^{u}\right) / \exp \left\{\tau_{i i} k_{i 2}\right\}:=k_{i 4}$; from Lemma 17, we have

$$
\begin{aligned}
\liminf _{t \rightarrow \infty} x_{i}(t) & \geq\left[\frac{r_{i}^{l}}{2}-k_{i 4} \exp \left\{M_{i 1}\right\}\right] l+\ln \frac{r_{i}^{l}}{2 k_{i 4}} \\
& :=m_{i 1} .
\end{aligned}
$$

From (1) and (75), there exists a constant $T_{7}>T_{6}$, such that, for $t>T_{7}$,

$$
\begin{aligned}
y_{i}^{\Delta}(t)+a_{i}(t) y_{i}(t)= & b_{i}(t) \exp \left\{x_{i}(t)\right\} \\
& +\beta_{i}(t) \exp \left\{x_{i}\left(t-\sigma_{i}\right)\right\} \\
\geq & \left(b_{i}^{l}+\beta_{i}^{l}\right) \exp \left\{m_{i 1}\right\} ;
\end{aligned}
$$

from Lemma 18, we have

$$
\liminf _{t \rightarrow \infty} y_{i}(t) \geq \frac{\left(b_{i}^{l}+\beta_{i}^{l}\right) \exp \left\{m_{i 1}\right\}}{a_{i}^{u}}:=m_{i 2} .
$$

From (66), (68), (75), and (77), we obtain

$$
\begin{aligned}
& m_{i 1} \leq \liminf _{t \rightarrow \infty} x_{i}(t) \leq \lim _{t \rightarrow \infty} \sup x_{i}(t) \leq M_{i 1}, \\
& m_{i 2} \leq \liminf _{t \rightarrow \infty} y_{i}(t) \leq \lim _{t \rightarrow \infty} \sup y_{i}(t) \leq M_{i 2} ;
\end{aligned}
$$

then the solutions for system (1) are uniformly ultimate bounded.

Therefore, the proof of Theorem 21 is completed.
Noticing the differences of Definitions 1 and 19, due to the exponential transformations in Section 1, when solutions of system (1) or (9) are uniformly ultimate bounded, systems (5) and (6) or (11) and (12) are permanent, and the contrary also holds true. Then we have the following remarks.

Remark 22. If $\mathbb{T}=\mathbb{Z}$, then Theorems 20 and 21 reduce to Theorems 6 and 4, respectively.

If $\mathbb{T}=\mathbb{R}$, we could get a new method to solve the continuous result from Theorem 20. Thus, our result is a well unification of continuous and discrete cases.

Remark 23. If $\mathbb{T}=\mathbb{R}$, then the result implies Theorem 3 .

It is obvious that the conditions of Theorem 21 are weaker than that in Theorem 2, and it includes the known results of $[14,16]$ on $\mathbb{R}$ or $\mathbb{Z}$, respectively. Thus, Theorem 21 is a well unification of Theorems 3 and 4 . And our result shows that the additional conditions in $[15,17]$ are not necessary.

\section{An Example}

In this section, we give a numerical example to support our main result. Let $\mathbb{T}=\bigcup_{k=0}^{\infty}[12 k+3,12 k+11]$; we assume

$$
\begin{array}{rl}
x^{\Delta}(t) & =(0.6+0.4 \cos (t)) \\
\cdot\left\{1-\frac{\exp \{x(t)\}}{1+0.5 \cos (t)}-0.01 y(t)\right\}, & \\
y^{\Delta}(t) & =-0.3 y(t)+(0.8+0.2 \sin (t)) \exp \{x(t)\}, \\
t & t \in \mathbb{T} ;
\end{array}
$$

it is easy to see that the conditions in Theorem 20 are verified. In this case, we know that

$$
\begin{aligned}
& x(12 k+3)=x(12 k-1)+4 \times r(12 k-1)\{1 \\
& \left.-\frac{\exp \{x(12 k-1)\}}{k(12 k-1)}-c(12 k-1) y(12 k-1)\right\}, \\
& y(12 k+3)=y(12 k-1)-4 \times a(12 k-1) y(12 k \\
& -1)+b(12 k-1) \exp \{x(12 k-1)\} ;
\end{aligned}
$$

here $k=1,2,3, \ldots$. By applying numerical analysis from Matlab, we obtain Figure 1. It is obvious that the solutions for system (79) are uniformly ultimate bounded. Our numerical simulation supports our theoretical findings (see Figure 1).

\section{Conclusion}

In this paper, we consider two systems with feedback control on time scales. It is shown that these systems contain the differential and difference systems as special cases. By constructing some differential inequalities on time scales, the uniform ultimate boundedness of solutions for these systems is obtained. Compared with some previous relative results, our results could unify the continuous and discrete cases sufficiently (see Theorems 3 and 4). This provides a new 


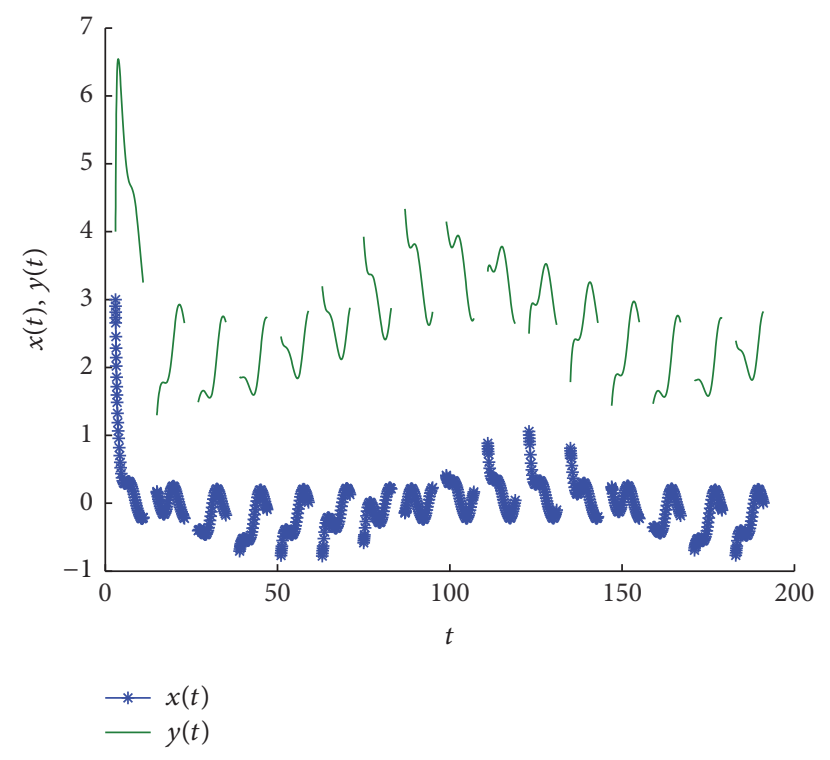

FIgURE 1: For the initial condition $x(0)=3$ and $y(0)=4$. “*” presents $x(t)$ and "-" presents $y(t)$.

method to get the permanence of ecosystems. Our results generalize and extend some known results. And a numerical example shows the verification of our main results.

\section{Competing Interests}

The authors declare that there is no conflict of interests regarding the publication of this paper.

\section{Authors' Contributions}

All authors contributed equally to the writing of this paper. All authors read and approved the final manuscript.

\section{Acknowledgments}

Research was supported by the National Natural Science Foundation of China (11201213 and 11371183), Natural Science Foundation of Shandong Province (ZR2015AM026 and ZR2013AM004), and the Project of Shandong Provincial Higher Educational Science and Technology (J15LI07).

\section{References}

[1] S. Hilger, Ein Maßkettenkalkül mit Anwendung auf Zentrumsmannigfaltigkeiten [Ph.D. thesis], Universität Würzburg, Würzburg, Germany, 1988.

[2] M. Bohner and A. Peterson, Dynamic Equations on Time Scales, Birkhäuser, Boston, Mass, USA, 2001.

[3] S. J. Schreiber and S. Patel, "Evolutionarily induced alternative states and coexistence in systems with apparent competition," Natural Resource Modeling, vol. 28, no. 4, pp. 475-496, 2015.

[4] A. Muhammadhaji, Z. Teng, and X. Abdurahman, "Permanence and extinction analysis for a delayed ratio-dependent cooperative system with stage structure," Afrika Matematika, vol. 25, no. 4, pp. 897-909, 2014.
[5] C. Zhao and L. Wang, "Convergence and permanence of a delayed Nicholson's Blowflies model with feedback control," Journal of Applied Mathematics and Computing, vol. 38, no. 1, pp. 407-415, 2012.

[6] A. Muhammadhaji and Z. D. Teng, "Permanence and extinction analysis for a periodic competing predator-prey system with stage structure," International Journal of Dynamics and Control, 2015.

[7] L.-L. Wang and Y.-H. Fan, "Permanence and existence of periodic solutions for a generalized system with feedback control," Applied Mathematics and Computation, vol. 216, no. 3, pp. 902-910, 2010.

[8] H. Hu, Z. Teng, and H. Jiang, "Permanence of the nonautonomous competitive systems with infinite delay and feedback controls," Nonlinear Analysis. Real World Applications. An International Multidisciplinary Journal, vol. 10, no. 4, pp. 2420 2433, 2009.

[9] J. Li, A. Zhao, and J. Yan, "The permanence and global attractivity of a Kolmogorov system with feedback controls," Nonlinear Analysis. Real World Applications. An International Multidisciplinary Journal, vol. 10, no. 1, pp. 506-518, 2009.

[10] F. Chen, "Permanence of a single species discrete model with feedback control and delay," Applied Mathematics Letters. An International Journal of Rapid Publication, vol. 20, no. 7, pp. 729733, 2007.

[11] Y.-H. Fan and L.-L. Wang, "Permanence for a discrete model with feedback control and delay," Discrete Dynamics in Nature and Society, vol. 2008, Article ID 945109, 8 pages, 2008.

[12] Y. Li and T. Zhang, "Permanence and almost periodic sequence solution for a discrete delay logistic equation with feedback control," Nonlinear Analysis: Real World Applications, vol. 12, no. 3, pp. 1850-1864, 2011.

[13] H. T. Zhang and F. D. Zhang, "Permanence of an $N$-species cooperation system with time delays and feedback controls on time scales," Journal of Applied Mathematics and Computing, vol. 46, no. 1-2, pp. 17-31, 2014.

[14] L. Chen and X. Xie, "Permanence of an N-species cooperation system with continuous time delays and feedback controls," Nonlinear Analysis. Real World Applications, vol. 12, no. 1, pp. 34-38, 2011.

[15] F. Chen, "Permanence of a discrete $N$-species cooperation system with time delays and feedback controls," Applied Mathematics and Computation, vol. 186, no. 1, pp. 23-29, 2007.

[16] Y. Li and T. Zhang, "Permanence of a discrete n-species cooperation system with time-varying delays and feedback controls," Mathematical and Computer Modelling, vol. 53, no. 56, pp. 1320-1330, 2011.

[17] F. Chen, X. Liao, and Z. Huang, "The dynamic behavior of Nspecies cooperation system with continuous time delays and feedback controls," Applied Mathematics and Computation, vol. 181, no. 2, pp. 803-815, 2006.

[18] Z. C. Jiang, W. B. Ma, and J. J. Wei, “Global Hopf bifurcation and permanence of a delayed SEIRS epidemic model," Mathematics and Computers in Simulation, vol. 122, pp. 35-54, 2016.

[19] K. Gopalsamy, Stability and Oscillations in Delay Differential Equations of Population Dynamics, Mathematics and its Applications, Kluwer Academic Publishers Group, Dordrecht, The Netherlands, 1992.

[20] Y.-H. Fan and L.-L. Wang, "Global asymptotical stability of a logistic model with feedback control," Nonlinear Analysis. Real World Applications. An International Multidisciplinary Journal, vol. 11, no. 4, pp. 2686-2697, 2010. 
[21] Y. Li and L. Zhu, "Existence of positive periodic solutions for difference equations with feedback control," Applied Mathematics Letters, vol. 18, no. 1, pp. 61-67, 2005.

[22] V. L. Kocic and G. Ladas, Global behavior of nonlinear difference equations of higher order with applications, vol. 256 of Mathematics and its Applications, Kluwer Academic Publishers Group, Dordrecht, Dordrecht, Netherlands, 1993. 


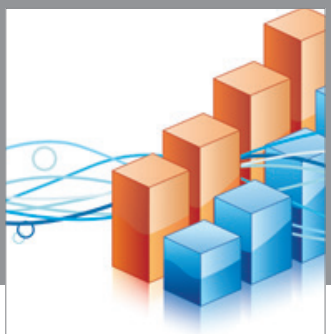

Advances in

Operations Research

vatem alat4

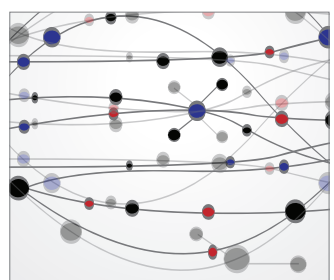

\section{The Scientific} World Journal
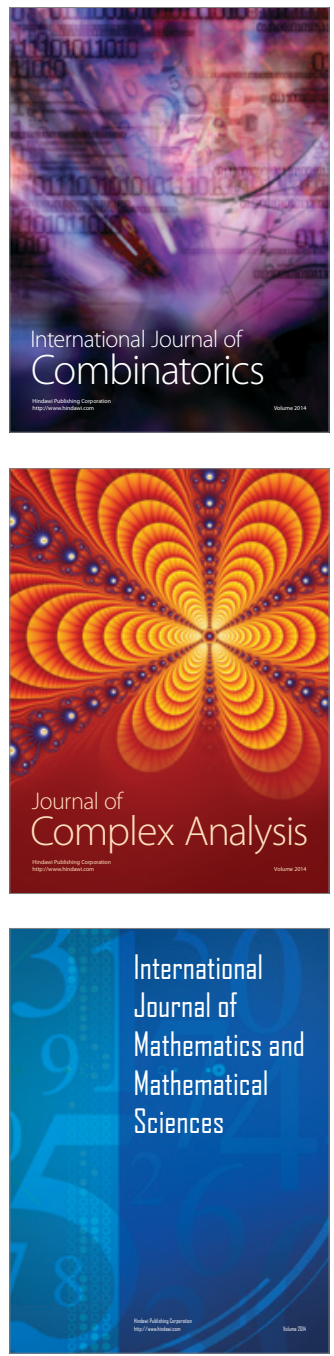
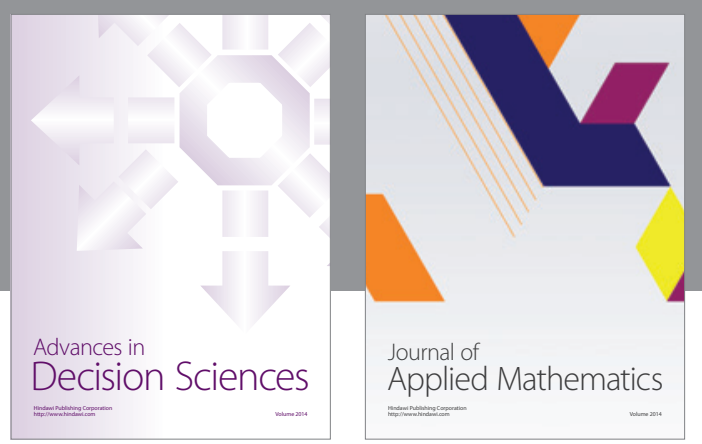

Algebra

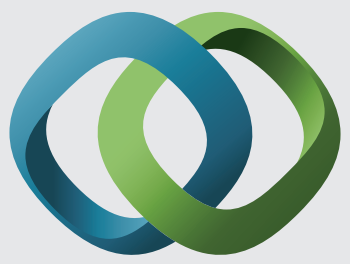

\section{Hindawi}

Submit your manuscripts at

https://www.hindawi.com
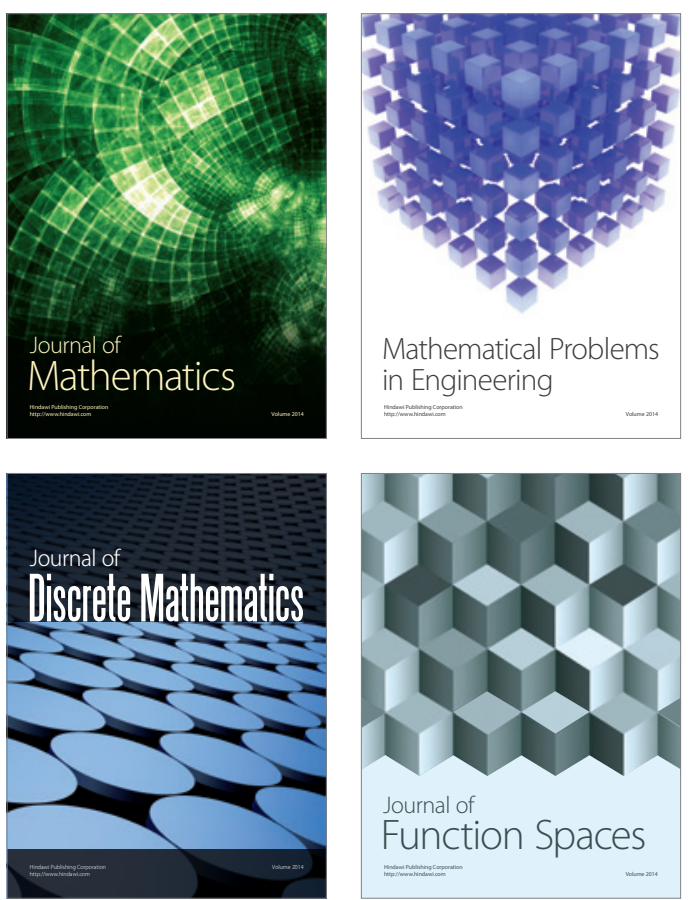

Mathematical Problems in Engineering
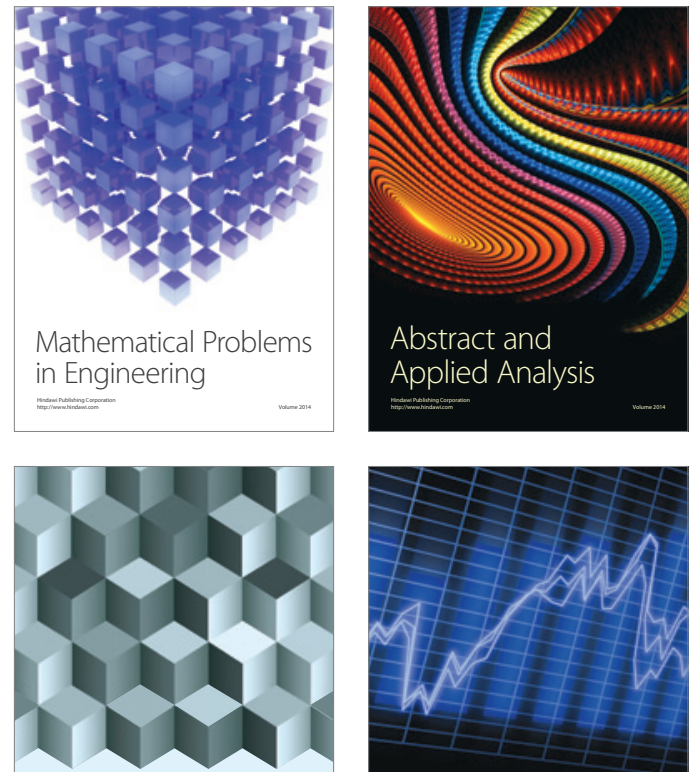

Journal of

Function Spaces

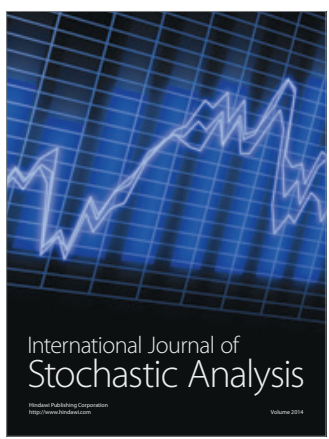

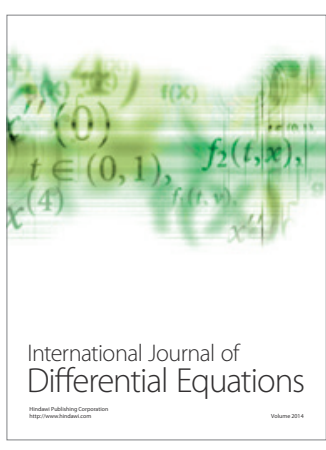
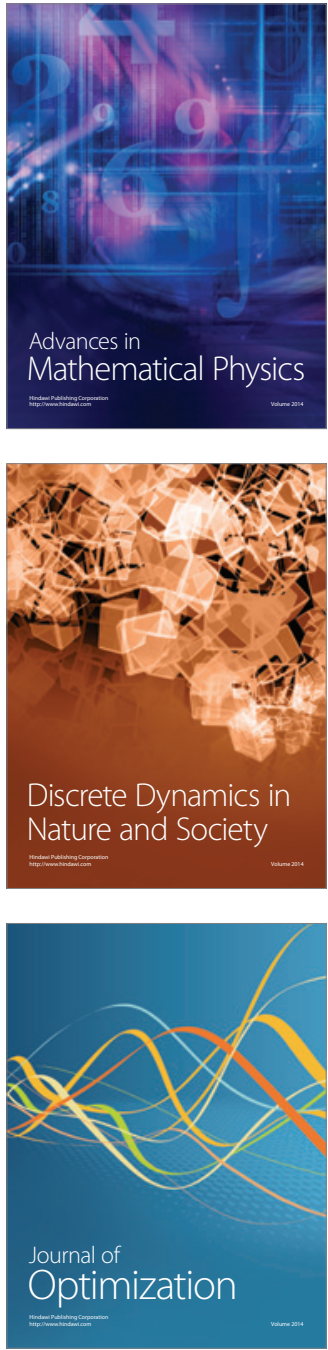